\title{
DETECÇÃO DE CONTAMINAÇÃO DE SOLO POR VINHAÇA ATRAVÉS DE ANÁLISE DE DADOS DE ELETRORRESISTIVIDADE
}

\author{
Juliana Igarashi da Cruz ${ }^{1}$, Rodrigo de Souza Portugal² ${ }^{2}$ M. Carmen Hernández Lucendo³ \\ Vagner Roberto Elis ${ }^{4}$, Sergio Junior da Silva Fachin ${ }^{5}$, Andrea T. Ustra ${ }^{6}$ e Welitom Rodrigues Borges ${ }^{7}$
}

Recebido em 31 outubro, 2007 / Aceito em 16 outubro, 2008

Received on October 31, 2007 / Accepted on October 16, 2008

\begin{abstract}
The vinasse, a waste produced in the proportion of 13 liters for each liter of alcohol. It has a high potential of polluting groundwater and superficial water resources, changes the soil behaviour and can also develop sanilization problems. This work aims to evaluate the efficiency of the DC-resistivity method in detecting and mapping anomalies caused by inappropriate disposal of vinasse in an inactive infiltration tank located at Sepé-Tiarajú settlement of landless agricultural laborers in the Ribeirão Preto region. Besides, as secondary goals, this work aims to characterize the type of anomaly residue as well as to diagnose its influence inside and outside of the limits of the tank. Eleven electrical resistivity tomography profiles were carried out with the dipole-dipole array, $10 \mathrm{~m}$ of dipoles length and 5 levels of investigation. The geophysical survey enabled us to conclude that the DC-resistivity method is appropriate for mapping the contamination plume caused by intense vinasse disposal and its influence. It enabled also to conclude that the contamination exceeds the tank limits. The vinasse influence can be characterized by low resistivity values between 10 Ohm.m and 90 Ohm.m and its behavior can be compared with the one of the chorume, which is also conductive.
\end{abstract}

Keywords: vinasse, DC-resistivity, soil contamination.

RESUMO. A vinhaça, resíduo gerado na proporção de 13 litros para cada litro de álcool produzido, possui um alto potencial poluidor dos recursos hídricos subterrâneos e superficiais, causa alterações no comportamento do solo e pode gerar problemas de salinização. Este trabalho objetiva avaliar a eficiência do método da eletrorresistividade para a detecção e mapeamento das anomalias causadas pela disposição inadequada de grandes quantidades de vinhaça em um antigo tanque de infiltração localizado no Assentamento Sepé-Tiarajú, Região de Ribeirão Preto - SP. 0 trabalho objetiva também caracterizar o tipo de anomalia inerente ao resíduo no local estudado e diagnosticar sua influência dentro e fora dos limites do tanque. Foram executados onze perfis de tomografia elétrica, arranjo dipolo-dipolo radial e espaçamento entre eletrodos de 10 metros e 5 níveis de investigação. Os ensaios geofísicos mostraram que o método da eletrorresistividade é apropriado para mapear as anomalias decorrentes da disposição de grandes quantidades de vinhaça no solo, apontar suas áreas de influência e concluir que a contaminação extrapola os limites do tanque. A influência da vinhaça pode ser caracterizada por baixos valores de resistividade entre 10 Ohm.m e 90 Ohm.m, podendo 0 seu comportamento ser comparado ao do chorume, que também é bastante condutivo.

Palavras-chave: vinhaça, eletrorresistividade, contaminação do solo

${ }_{1}^{1}$ ós-Graduada pelo Instituto de Geociências, Universidade Estadual de Campinas, Universidad Complutense de Madrid, Av. João Pandiá Calógeras, 51, Cidade Universitária Zeferino Vaz s/n, 13083-870 Campinas, SP, Brasil. Tel.: +55(19) 3521-5156 /+55(11) 2421-5342; Fax: +55(19) 3289-1562 - E-mail: juigarashi@gmail.com 2 Departamento de Geologia e Recursos Naturais, Instituto de Geociências, Universidade Estadual de Campinas, João Pandiá Calógeras, 51, Cidade Universitária Zeferino Vaz s/n, 13083-870 Campinas, SP, Brasil. Tel.: +55(19) 3521-5158; Fax: +55(19) 3289-1562 - E-mail: portugal@ige.unicamp.br

${ }^{3}$ Departamento de Física de la Tierra I, Facultad de Ciencias Físicas, Universidad Complutense de Madrid, Ciudad Universitaria, 28.040, Madrid, Espanha. Tel.: +34(91) 394-4391; Fax: +34(91) 394-4398 - E-mail: geofmc@fis.ucm.es

${ }^{4}$ IAG/USP, Departamento de Geofísica, Rua do Matão, 1.226, Cidade Universitária, Butantã, 05508-090 São Paulo, SP, Brasil. Tel.: +55(11) 3091-4749; Fax: +55(11) 3091-5034 - E-mail: vagnelis@iag.usp.br

${ }^{5}$ Curso de Pós-Graduação em Geofísica, Instituto de Astronomia, Geofísica e Ciências Atmostéricas, Rua do Matão, 1.226, Cidade Universitária, 05508-090 São Paulo, SP, Brasil. Tel.: +55(11) 3091-2792 - E-mail: sergiustga@gmail.com

${ }^{6}$ Curso de Pós-Graduação em Geofísica, IAG-USP, Rua do Matão, 1.226, Cidade Universitária, 05508-090 São Paulo, SP, Brasil. Tel.: +55 (11) $3091-4753$; Fax: +55 (11) 3091-5034-E-mail: andreaustra@iag.usp.br

7Laboratório de Geofísica Computacional, IMECC/UNICAMP, Rua Sérgio Buarque de Holanda, 651, Cidade Universitária Zeferino Vaz s/n, 13083-870 Campinas, SP, Brasil. Tel.: +55(19) 3521 5968; Fax: +55(11) 3289-5766 - E-mail: welitom.geologo@gmail.com 


\section{INTRODUÇÃO E OBJETIVOS}

Atualmente, a busca por combustíveis alternativos vem gerando uma considerável demanda da commodity etanol, cujo importante produtor é 0 Brasil. Segundo Macedo (2007), 425 milhões de toneladas de cana foram processadas no Brasil em 2006 produzindo 30 milhões de toneladas de açúcar e 17 milhões de metros cúbicos de etanol.

Com 0 crescimento das vendas dos veículos biocombustíveis e da competitividade do etanol frente à gasolina, a expectativa é de que em 2012/2013 sejam processados em torno de 700 milhões de toneladas de cana, produzindo 36 bilhões de litros de álcool e 39 bilhões de toneladas de açúcar (Unica, 2007). Segundo Cabrini \& Marjotta-Maistro (2007), a quantidade de álcool exportada pelo Brasil no período de 2001 a 2005 cresceu cerca de $652 \%$.

0 aumento da demanda e da produção de etanol intensifica as preocupações com 0 meio-ambiente, em especial pelos problemas causados pelos resíduos de sua produção. A vinhaça é o principal efluente das destilarias de álcool e é produzida, em média, à razão de 13 litros por litro de álcool destilado (Ludovice, 1997).

A vinhaça também é conhecida como vinhoto, calda, restilo, garapão, vinhote, caxixi, mosto, still bottoms, slops, vinasse, dunder, stillage, cachaza, entre outras denominações, e pode alterar as propriedades físicas e químicas do solo e contaminar os recursos hídricos superficiais e subterrâneos se disposta de forma inadequada (Freire, 2000).

A vinhaça é um líquido de cor marrom escuro, de natureza ácida, elevada Demanda Bioquímica de Oxigênio (DBO), corrosivo e altamente poluidor. É composta de $93 \%$ de água e $7 \%$ de sólidos, dentre os quais 75\% são compostos por matéria orgânica e 25\% de fração mineral (Ludovice, 1997).

A vinhaça caracteriza-se pelo alto conteúdo de matéria orgânica e potássio, quantidades apreciáveis de nitrogênio, fósforo, cálcio, magnésio e sulfato e quantidades menores de zinco, ferro, manganês e cobre. 0 pH normalmente é acido e a condutividade elétrica é bastante elevada (Hassuda, 1989).

Grandes doses de vinhaça podem ter um impacto severo no solo e nas águas superficias e subterrâneas. Gloeden (1994) estudando a dinâmica dos constituintes da vinhaça nas zonas saturada e não saturada, concluiu que o cloro, 0 carbono orgânico, 0 amônio e 0 nitrogênio orgânico aparecem como elementos que impõem risco à qualidade da água subterrânea. A infiltração da vinhaça na água subterrânea prejudica sua potabilidade pela transferência de altas concentrações de amônia, magnésio, alumínio, ferro, manganês, cloreto e matéria orgânica para a água subterrânea (Hassuda, 1989).

Acrescentando-se a este contexto a necessidade de buscar formas viáveis de detecção e avaliação de contaminações no meio geradas pela disposição inadequada de resíduos urbanos e industriais, os métodos geofísicos apresentam-se como uma importante ferramenta de investigação indireta da subsuperfície, nãodestrutiva e de baixo custo quando comparada com as técnicas diretas de investigação (Reynolds, 1997).

Determinados métodos geofísicos permitem obter dados contínuos em grandes áreas de cobertura, minimizando a necessidade de dados diretos e específicos, possibilitando uma análise temporal e espacial detalhada (Reynolds, 1997) e permitindo também a identificação mais precisa das variações laterais decorrentes de mudanças litológicas ou variações originadas pela presença de resíduos.

Especificamente na avaliação da contaminação na superfície terrestre, os métodos geofísicos são bastante utilizados para a Iocalização de valas contendo resíduos, investigação da contaminação disseminada no solo e nas águas subterrâneas, detecção de tambores e tanques enterrados e determinação de vazamentos em tanques ou dutos (Cetesb, 2004).

0 método da eletrorresistividade baseia-se no contraste de resistividade elétrica dos materiais em uma dada área de amostragem e sua aplicação em estudos ambientais é possível em conseqüência do caráter mais resistivo ou mais condutivo de um dado contaminante frente ao terreno.

Estes contrastes dão lugar às anomalias, que podem indicar a presença de contaminação em subsuperfície, oferecendo subsídios para diagnosticar, monitorar a propagação de contaminantes e acompanhar a recuperação de uma área contaminada (Cetesb, 2004).

A aplicação de métodos elétricos para estudos ambientais é amplamente relatada na literatura, podendo-se citar Mendes (1987), Hassuda (1989), Vogelsang (1995), Reynolds (1997), Bernstone et al. (2000); Meju (2000); Aristodemou \& ThomasBetts (2000); Ahmed \& Sulaiman (2001); Moura \& Malagutti Filho (2003). 0 presente artigo objetiva avaliar a eficiência do método da eletrorresistividade para a detecção e mapeamento das anomalias causadas pela disposição inadequada de grandes quantidades de vinhaça em um antigo tanque de infiltração localizado no Assentamento Sepé-Tiarajú, Região de Ribeirão Preto $\mathrm{SP}$, bem como caracterizar o tipo de anomalia inerente ao resíduo no local estudado e diagnosticar sua influência dentro e fora dos limites do tanque.

\section{CARACTERIZAÇÃO DA ÁREA DE ESTUDO Localização e descrição do tanque desativado}

0 Assentamento Sepé Tiarajú localiza-se na região de Ribeirão Preto, nos Municípios de Serrana e Serra Azul - SP (Fig. 1), entre 0 km 28 e 0 km 31,4 da Rodovia 333 - Abraão Assed. 

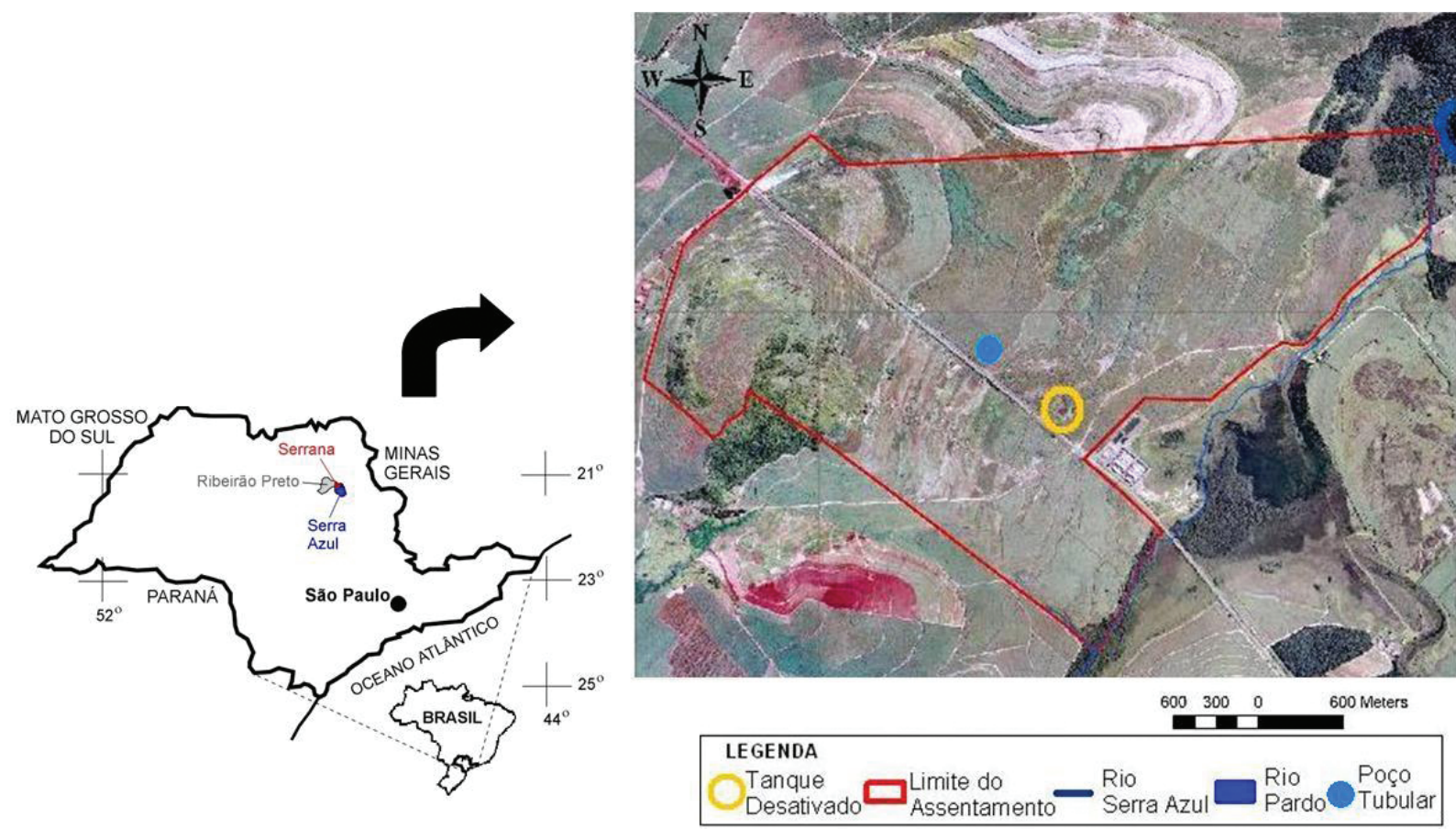

Figura 1 - Localização dos Municípios de Serrana e Serra Azul, região de Ribeirão Preto - SP e imagem do Assentamento Sepé Tiarajú.

0 tanque de vinhaça desativado, alvo da pesquisa, tinha a função de servir de centro de distribuição e armazenagem do resíduo, que era transferido do tanque para os caminhões e transportado para áreas mais distantes, onde era então utilizado como adubo através da fertirrigação dos canaviais.

0 tanque utilizado para a armazenagem do vinhoto (Fig. 2) é um quadrado cujo lado externo possui 90 metros, e a parte interna, aproximadamente 70 metros. Não há dados concretos sobre a profundidade real do tanque em sua construção, atualmente, 0 volume do tanque desativado não ocupado com resíduos é de aproximadamente 19.300 metros cúbicos.

Um poço tubular localizado a cerca de 600 metros do tanque indica o nível estático naquele ponto (Fig. 1) a 52 metros de profundidade.

0 tanque não possui qualquer tipo de manta geoimpermeabilizante, tendo em vista que na época de sua instalação não havia as obrigações legislativas e as tecnologias existentes atualmente, dessa forma, a sua impermeabilização é resultante apenas da presença natural de argila no solo.

\section{Geologia}

A área de estudos está localizada nos domínios da bacia sedimentar do Paraná, ocorrendo localmente as formações Botucatu, Pirambóia e Serra Geral. 0 Relatório Ambiental Preliminar (RAP) solicitado ao Departamento de Estradas de Rodagem do Estado de São Paulo (DER/SP, 2006), em estudo obrigatório requerido para a duplicação da pista da Rodovia SP-333 - Abrão Assed do km 33,8 ao km 54,8, portanto a cerca de $2 \mathrm{~km}$ do Assentamento Sepé-Tiarajú, descreve que a região é constituída pelos derrames basálticos da Formação Serra Geral, rochas sedimentares das Formações Botucatu e Pirambóia, sedimentos continentais terciários e sedimentos aluvionais quaternários.

Quanto à hidrogeologia do local, o mesmo estudo supracitado considera que a maior parte daquela área de estudo está sobre um aqǘfero fissurado, representado pelos basaltos da Formação Serra Geral, e em uma parcela inferior, sobre o Aqǘfero Guarani, representado pelos arenitos da Formação Botucatu e coberturas cenozóicas.

Um furo de sondagem realizado dentro do tanque desativado mostra a presença de 0,2 metros de um solo argiloso orgânico, 0,25 metros de um solo argiloso de coloração vermelha, sobrepostos a um solo argiloso de coloração marrom escuro com presença de resíduos até a profundidade de 3,7 metros. Outros dois furos de sondagem realizados fora do tanque não apresentam variações no perfil, podendo ser descritos como solo argiloso de coloração avermelhada atéa profundidade de 3 metros (Fig. 3).

\section{METODOLOGIA}

\section{Revisão teórica}

0 método da eletrorresistividade consiste em medir as resistividades do meio pela aplicação de corrente elétrica. A injeção de corrente é feita por meio de dois eletrodos de corrente e a dife- 


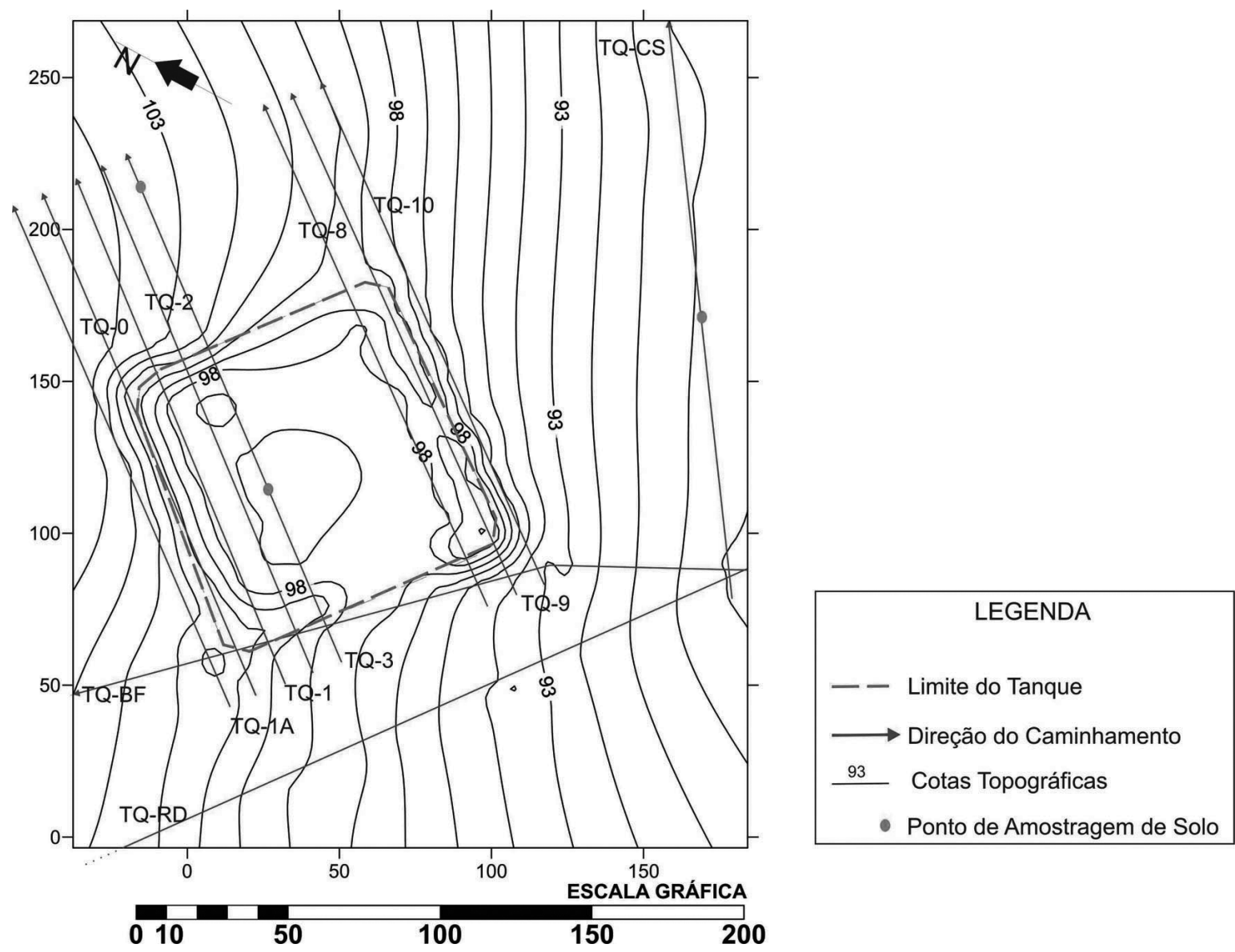

Figura 2 - Localização das linhas de tomografia elétrica.

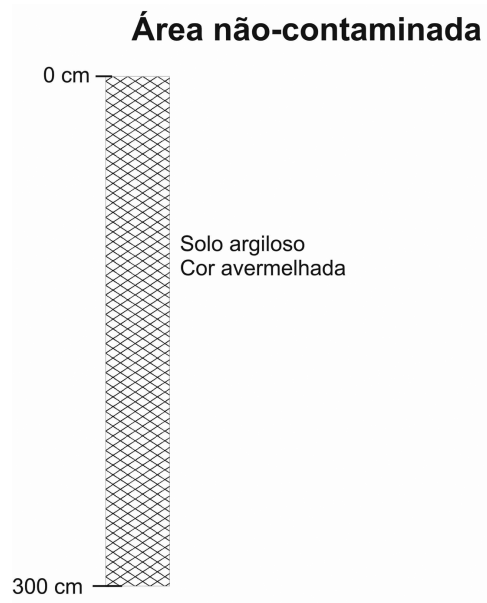

Tanque de Infiltração Desativado

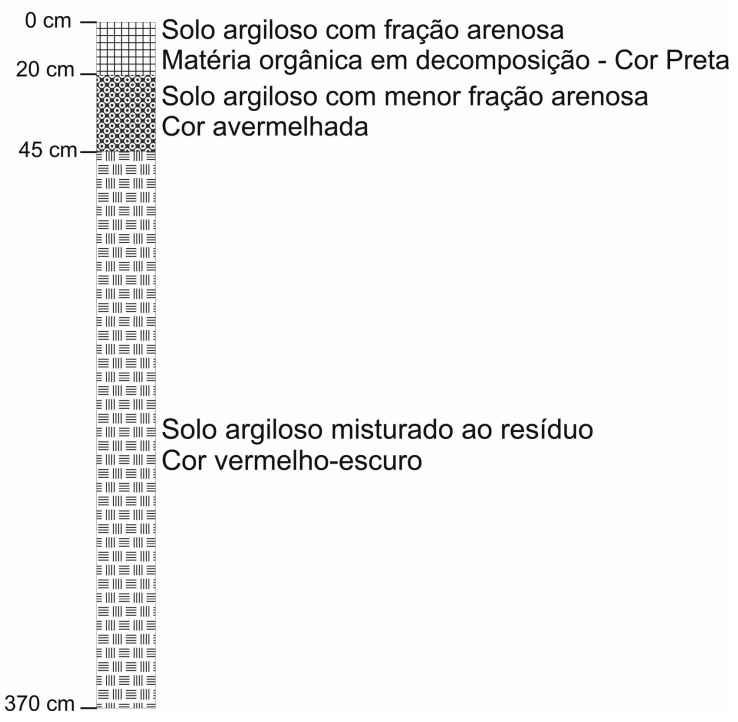

Figura 3 - Descrição geológica do tanque desativado. 
rença de potencial é mensurada por dois eletrodos de potencial. Os eletrodos são dispostos na superfície do solo segundo diversos arranjos e os dados adquiridos são relacionados com as propriedades elétricas da estruturação geológica em subsuperfície e com a resposta de cada material ao fluxo de uma corrente elétrica.

Para solos, a resistividade elétrica depende de muitos fatores, como a porosidade, a resistividade elétrica do fluído contido nos poros, a composição do solo, grau de saturação, orientação e forma das partículas e estrutura do poro (Keller \& Frischknecht, 1966; Parkhomenko, 1967; Abu-Hassanein et al., 1996).

A resistividade dos solos e das rochas tende a diminuir com 0 aumento da umidade e do volume de sólidos dissolvidos na água intersticial. A resistividade de sedimentos não-saturados é muito mais alta que a resistividade dos mesmos sedimentos em condições saturadas em água (McNeill, 1980).

As rochas metamórficas e ígneas possuem tipicamente altos valores de resistividade. Rochas sedimentares, que em geral são mais porosas e possuem maior conteúdo de água, tendem a ter valores de resistividade mais baixos (Loke, 2000).

As rochas e os solos argilosos tendem a ser menos resistivos que seus equivalentes arenosos, uma vez que a superfície de contato por unidade de volume da argila é muito grande e muitos íons são adsorvidos pela sua superfície. Quando a argila é imersa em um fluído, os íons adsorvidos podem se dissociar das partículas de argila e se tornar disponíveis, contribuindo para 0 aumento da condutividade (McNeill, 1980).

A relação entre água e solo na zona não saturada é definida pelo processo resultante das forças matriciais, que incluem a capilaridade e a adsorção, criando uma fina camada de água em torno das partículas dos sedimentos. A presença de matéria orgânica intensifica esse processo e aumenta a quantidade de água retida pelo solo (Van Dam, 2001). 0 conhecimento destes fatores é importante, pois o teor de umidade e a quantidade de sais dissolvidos na superfície rasa do tanque são significativos.

\section{Ensaios}

Para cumprir os objetivos foram realizadas onze linhas de tomografia elétrica utilizando-se 0 arranjo dipolo-dipolo radial e espaçamento de 10 metros entre os eletrodos. Foram utilizados os equipamentos Syscal R2 e Elrec Pro, ambos fabricados pela empresa francesa IRIS Instruments e de propriedade do Departamento de Geofísica do IAG/USP. 0 equipamento Syscal R2 realizou a função de módulo de transmissão de corrente elétrica e o Elrec PRO operou como módulo de medição de resistência elétrica.

Todas as linhas, exceto as linhas nomeadas como TQ-BF e TQ-RD, foram posicionadas perpendicularmente ao sentido provável do fluxo subterrâneo, sugerido pela topografia como direção sudeste. As linhas localizadas no intervalo TQ-00 a TQ10 foram planejadas para cobrir toda a área do tanque, segundo espaçamentos de 10 metros entre as linhas, no entanto, em decorrência da presença de uma lâmina de água em uma parte do tanque, não foi possível cumprir este planejamento.

As linhas TQ-01, TQ-02, TQ-03 e TQ-08, todas com 180 metros de comprimento, cobrem também a área interna do tanque de infiltração e permitem visualizar o contraste de condutividade dentro e fora do mesmo. A nomeação das linhas foi feita de forma que o número posterior à sigla $\mathrm{TQ}$ indique a distância em metros da linha referência TQ-00 multiplicado por um fator 10 (Fig. 2). Estas linhas foram planejadas com o objetivo específico de verificar a presença de anomalias decorrentes da disposição direta de vinhaça no solo e, por conseqüência, avaliar a eficiência do método da eletrorresistividade.

As linhas TQ-10 e TQ-BF foram realizadas imediatamente ao final topográfico das bordas do tanque e tiveram por objetivo verificar se as anomalias ultrapassam os limites da cava.

As linhas TQ-1A, TQ-CS e TQ-RD, respectivamente com 180 metros, 190 metros e 300 metros de comprimento, foram feitas com 0 objetivo de delimitar a região anômala, já que não era esperado qualquer tipo de anomalia nestas áreas.

Para melhor descrição e compreensão da área de pesquisa foi realizado um levantamento topográfico utilizando-se 0 equipamento Estação Total - TCR 305, produzido pela Leica Geosystems, cobrindo-se toda a área de pesquisa. A base da estação foi colocada na posição 20 metros da linha TQ-09, que permitia visualizar toda a área. Os dados topográficos são apresentados na Figura 2.

Os dados de resistividade aparente adquiridos em campo foram submetidos à rotina de inversão implementada pelo software comercial RES2DINV com o objetivo de obter modelos de resistividades reais da subsuperfície. A manipulação gráfica dos dados topográficos foi realizada com 0 software SURFER 8.0 (Golden Software, 2006).

\section{RESULTADOS}

Os dados correspondentes às linhas TQ-01, TQ-02, TQ-03 e TQ08, que atravessam a área interna do tanque, mostram a influência do resíduo e permitem demarcar os limites do mesmo (Figs. 6 , 7,8 e 9). A influência do resíduo é sugerida pelos baixos valores de resistividade elétrica (entre 10 0hm.m e 50 0hm.m) e, portanto, pelos altos valores de condutividade elétrica resultantes da grande quantidade de sais presentes na vinhaça.

Nestas linhas, a parte interna do tanque está compreendida entre as posições 30 metros e 100 metros das seções. As anomalias de condutividade podem ser notadas da superfície a profundidade máxima de 13 metros. Os limites da cava podem 


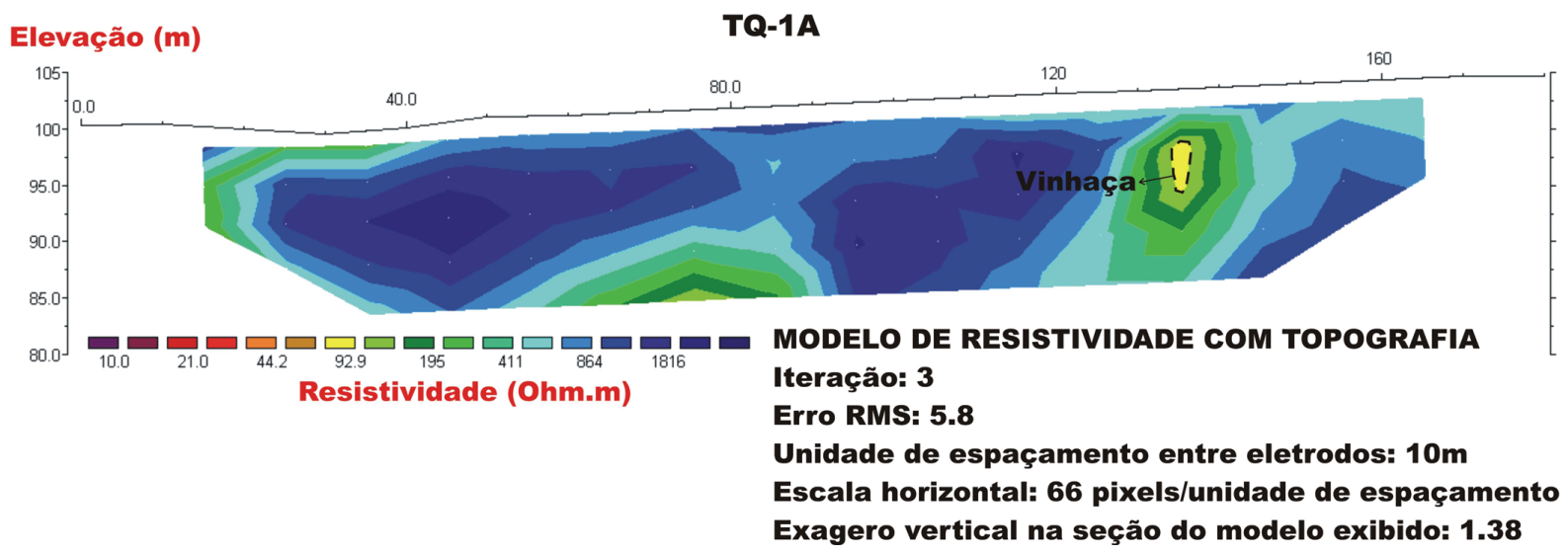

Figura 4 - Linha $T Q-1 A$, localizada 10 metros a montante do tanque.

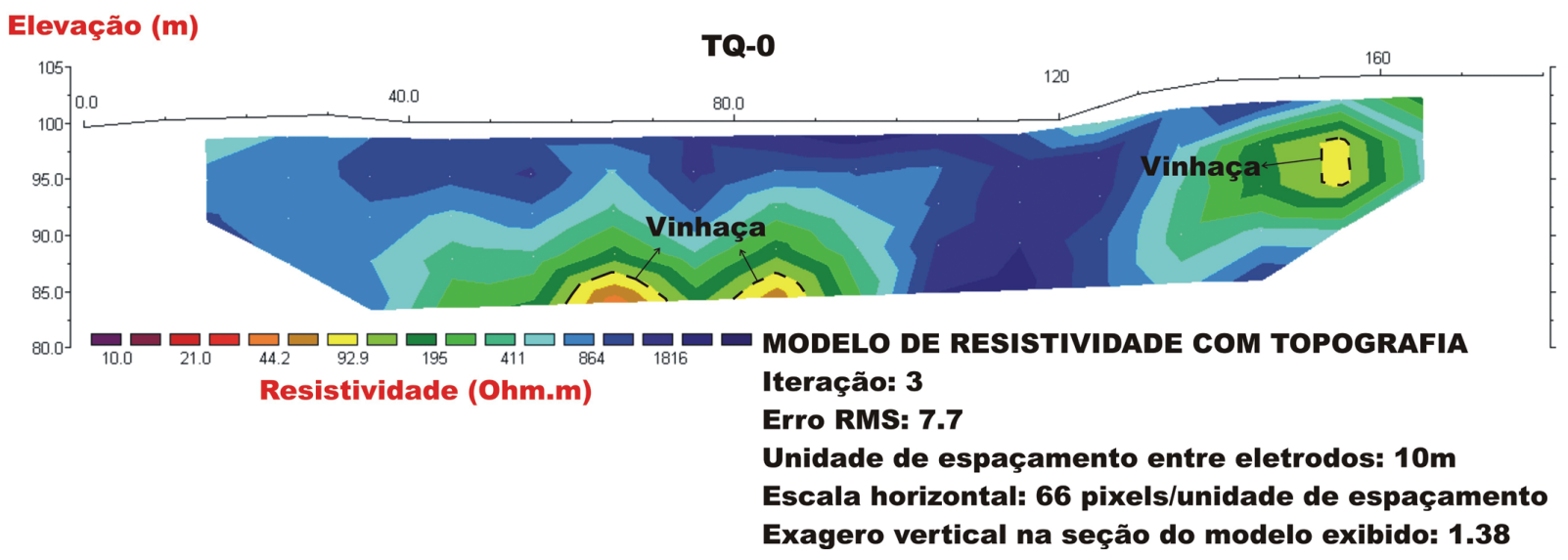

Figura 5 - Linha TQ-00, localizada a 10 metros da linha TQ-1A.

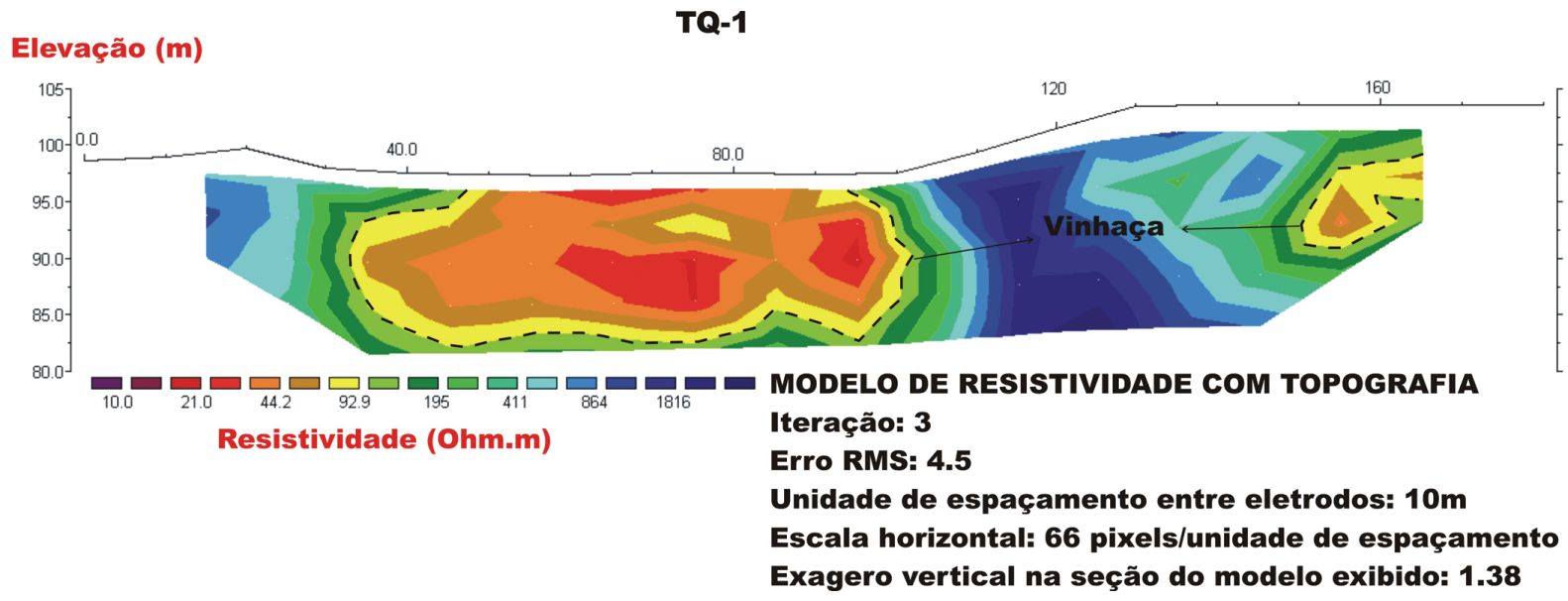

Figura 6 - Linha TQ-01, localizada dentro do tanque, a 10 metros da linha TQ-00. 


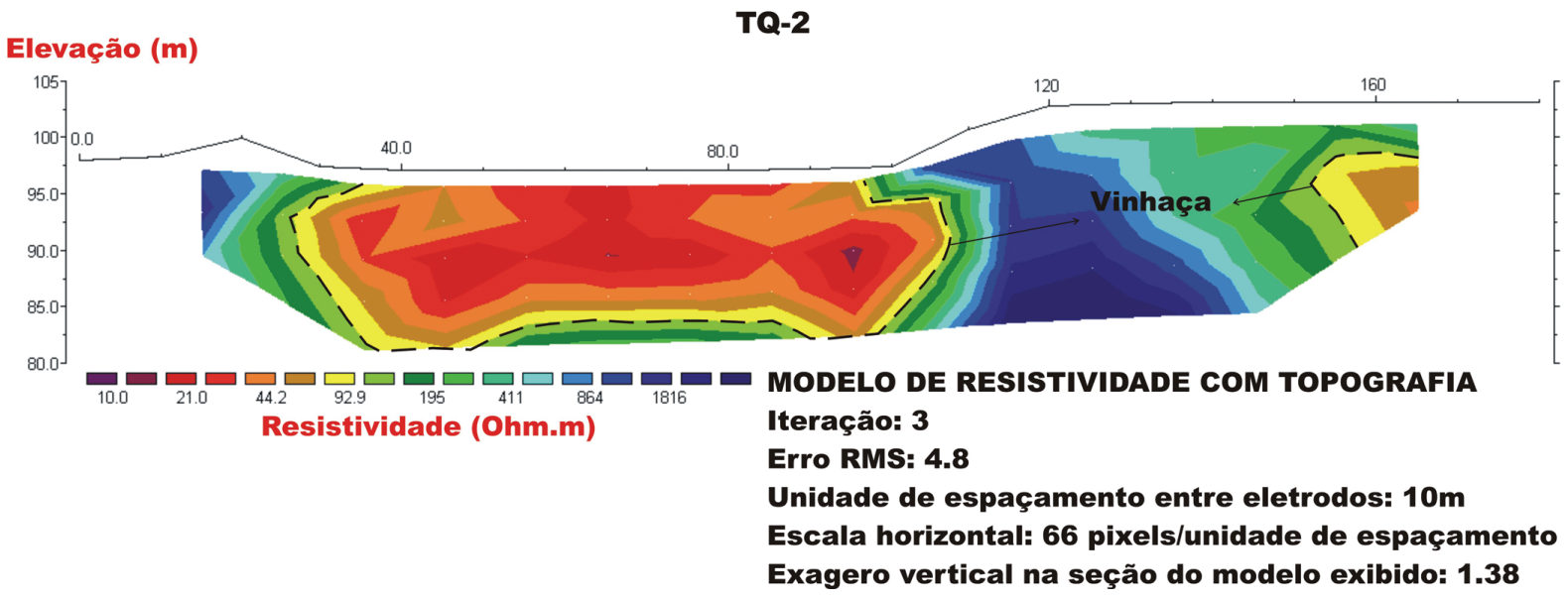

Figura 7 - Linha TQ-02 dentro da cava, a 10 metros da linha TQ-01.

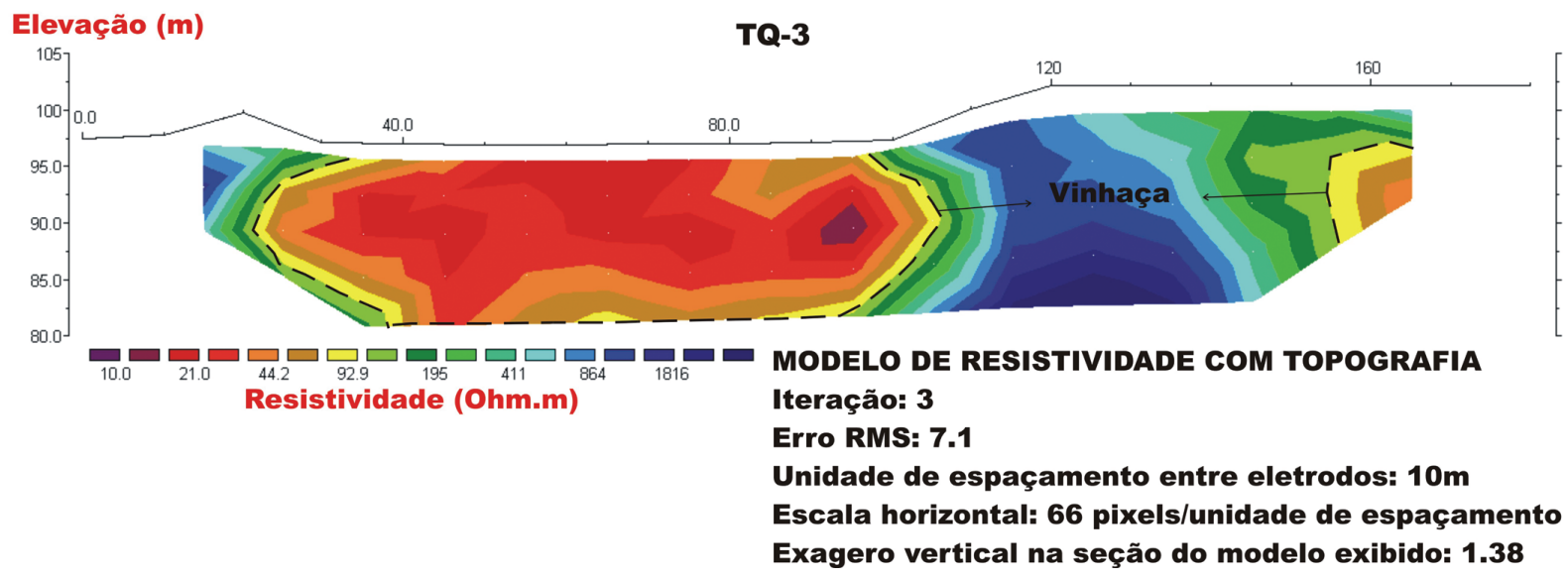

Figura 8 - Linha TQ-03 dentro da cava, a 10 metros da linha TQ-02.

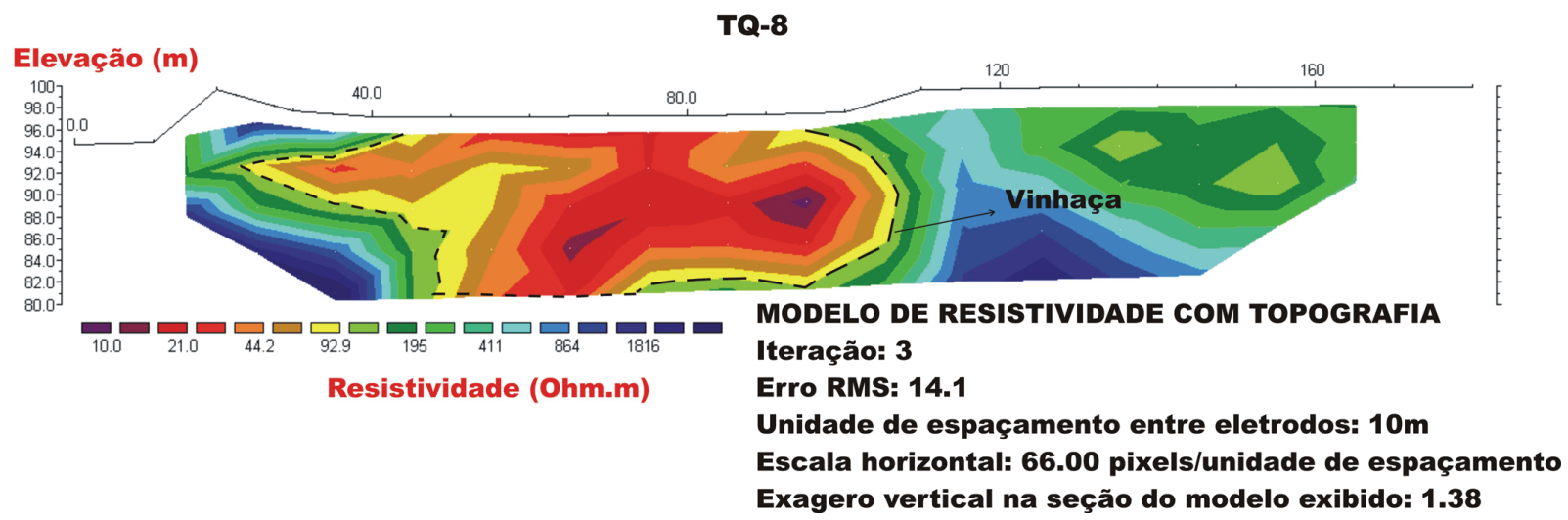

Figura 9 - Linha TQ-08, na borda interna do tanque, a 50 metros da linha TQ-03. 
ser identificados pelo aumento nos valores de resistividade (superiores a 130 Ohm.m).

Os dados da linha TQ-00 indicam alguma influência do tanque de vinhaça a aproximadamente 15 metros de profundidade (elevação 85 metros) entre as posições 50 metros e 90 metros (Fig. 5). A linha TQ-1A foi realizada a montante e a 10 metros da linha TQ-00 e sugere uma área anômala relacionada à vinhaça em torno das posições 55 metros e 85 metros e elevação 97 metros com valores de resistividade superiores a 150 Ohm.m (Fig. 4). Deve ser ressaltado que essa influência é muito baixa, haja vista os valores de resistividade relativamente altos comparados com os valores obtidos dentro do tanque, em torno de 15 Ohm.m.

Os dados da linha TQ-09, localizada sobre a borda da cava, à jusante do tanque, mostram ainda forte influência do tanque de vinhaça (Fig. 10). Nota-se baixos valores de resistividade (inferiores a 25 0hm.m) entre as posições 50 metros e 105 metros (elevações 93 metros e 81 metros).

Os dados da linha TQ-10 sugerem a influência direta do contaminante disposto no tanque, como pode ser observado pelos valores de resistividades em torno de 50 0hm.m a aproximadamente 12 metros de profundidade (elevação 87 metros) entre as posições 55 metros e 100 metros.

A linha seguinte, TQ-CS, de 190 metros de extensão e topografia relativamente constante, não apresenta dados anômalos de baixa resistividade, sugerindo que não há mais qualquer tipo de influência do contaminante (Fig. 12).

A linha TQ-BF corta todas as outras linhas e possui 220 metros de comprimento. Como TQ-10, esta linha segue 0 final da borda lateral externa do tanque e acompanha 0 declive natural do terreno.

Similarmente à linha TQ-10, os dados da linha TQ-BF sugerem uma influência direta do tanque através de uma anomalia de condutividade (em torno de 60 Ohm.m) entre as posições 80 metros e 110 metros a aproximadamente 12 metros de profundidade (elevação 87 metros) (Fig. 13).

A linha TQ-RD, de 300 metros de comprimento, foi feita seguindo a estrada de terra, próxima à TQ-BF. Esta linha, como TQ-CS, tem por finalidade delimitar a área de influência do contaminante, estabelecendo os contrastes de resistividade elétrica entre as zonas anômalas e as não anômalas. As altas resistividades encontradas nesta linha (superiores, em grande parte, a 1000 Ohm.m) cumprem este objetivo (Fig. 14).

Todas as linhas entre TQ-00 e TQ-10, incluindo a linha TQ$1 \mathrm{~A}$, apresentaram valores de resistividade entre $40 \mathrm{0hm} . \mathrm{m} \mathrm{e}$ 300 Ohm.m no canto superior direito, aproximadamente entre as posições 130 metros e 165 metros, a posição destas anomalias corresponde às curvas de nível (Fig. 2), responsáveis por alimentar o tanque com a vinhaça. Desta forma, essas anomalias de condutividade também podem ser atribuídas à vinhaça.

A linha TQ-BF apresentou uma anomalia de condutividade com valores entre 50 Ohm.m e 100 Ohm.m entre as posições 180 metros e 200 metros em torno de 8 metros de profundidade. Esta anomalia possivelmente pode ser atribuída à área onde os caminhões costumavam ser abastecidos com vinhaça.

Em complemento a essas anomalias condutivas, ressalta-se a presença de valores menores de resistividade (entre 150 Ohm.m e 500 Ohm.m) no canto esquerdo da linha TQ-CS (entre 15 metros e 30 metros) e no canto direito da linha TQ-RD (entre 220 metros e 285 metros), ponto de intersecção entre esses três perfis. Embora esses valores sejam bastante altos, ainda são significativamente menores que aqueles apresentados no resto dos três perfis mencionados.

A zona saturada e a base da área de influência da vinhaça não foram detectadas em nenhum dos ensaios.

Os baixos valores de resistividade relacionados às zonas de influência da vinhaça podem ser atribuídos à grande quantidade de sais presentes no resíduo e à retenção de água em solo argiloso explicada pelas forças matriciais. Dentro do tanque, a quantidade de água retida é maior, já que além de possuir maior concentração de material orgânico, o tanque ainda serve como captador de água de uma parte do assentamento, que chega até o tanque através das curvas de nível (da mesma forma que a vinhaça chegava até o tanque quando este ainda estava em uso). Esta última parte explica a diferença de umidade dentro e fora do tanque.

Os altos valores de resistividade encontrados nos perfis que cruzam o tanque e nas linhas TQ-CS e TQ-RD sugerem ausência da influência da vinhaça. Como o pacote de solo estudado é essencialmente composto por argila, não eram esperados valores tão altos de resistividade para as áreas sem influência da vinhaça.

Os valores encontrados para argila parecem demasiadamente altos, mas são bastante coerentes. Todas as linhas apresentaram 0 mesmo padrão de valores: resistividades altas nos locais sem influência da vinhaça e resistividades baixas dentro do tanque e nas zonas de influência do contaminante. Os altos valores de resistividade podem ser atribuídos a algumas possíveis explicações:

- Os altos valores encontrados nas bordas do tanque (principalmente entre as posições 100 metros e 120 metros) podem ser atribuídos a uma possível compactação abaixo do teor ótimo de umidade;

- Os altos valores encontrados fora das bordas do tanque, em especial nas linhas TQ-CS e TQ-RD podem ser atribuídos a um solo argiloso seco, e sem compactação, onde o espaço preenchido com ar, que separa os sedimentos, é suficiente para que não ocorra condução de corrente entre as partículas. 


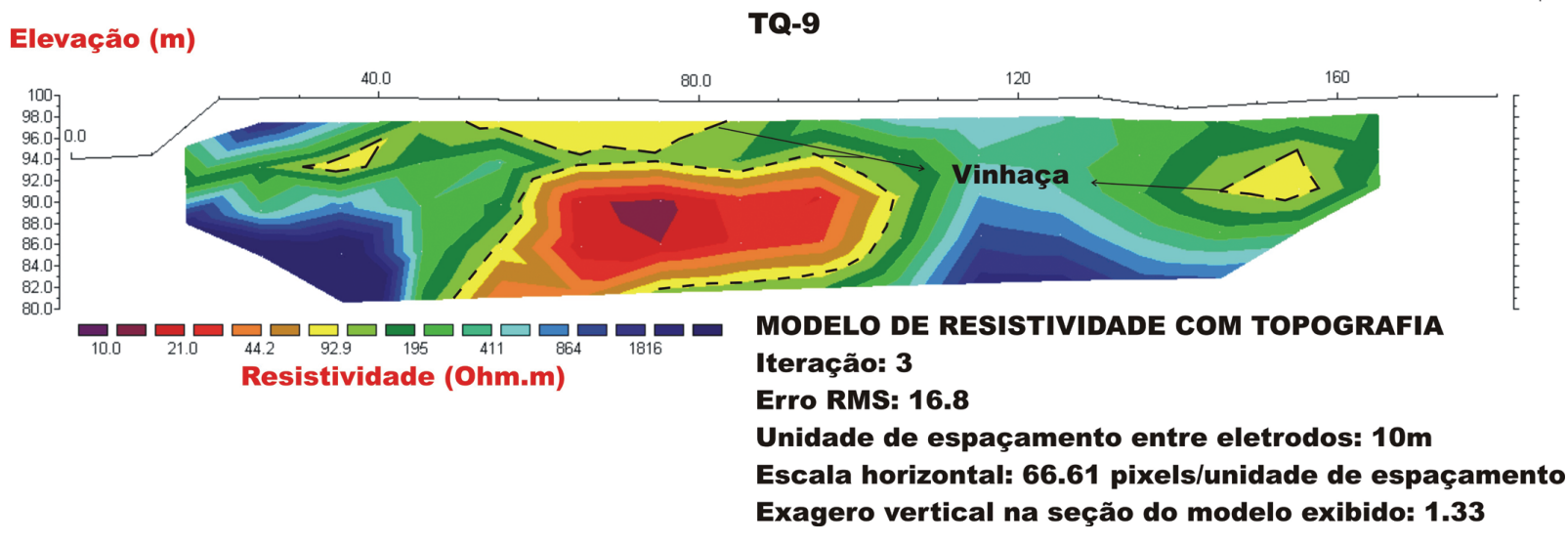

Figura 10 - Linha TQ-09, sobre a borda do tanque, a 10 metros da linha TQ-08.

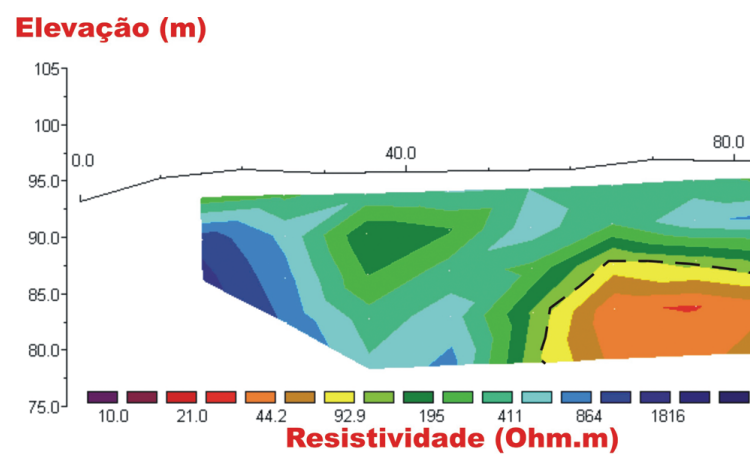

\section{TQ-10}

\section{Iteração: 3}

Erro RMS: 3.4

Unidade de espaçamento entre eletrodos: $10 \mathrm{~m}$ Escala horizontal: 66.56 pixels/unidade de espaçamento Exagero vertical na seção do modelo exibido: 1.37

Figura 11 - Linha TQ-10, localizada 10 metros após a linha TQ-09.

\section{TQ-CS}

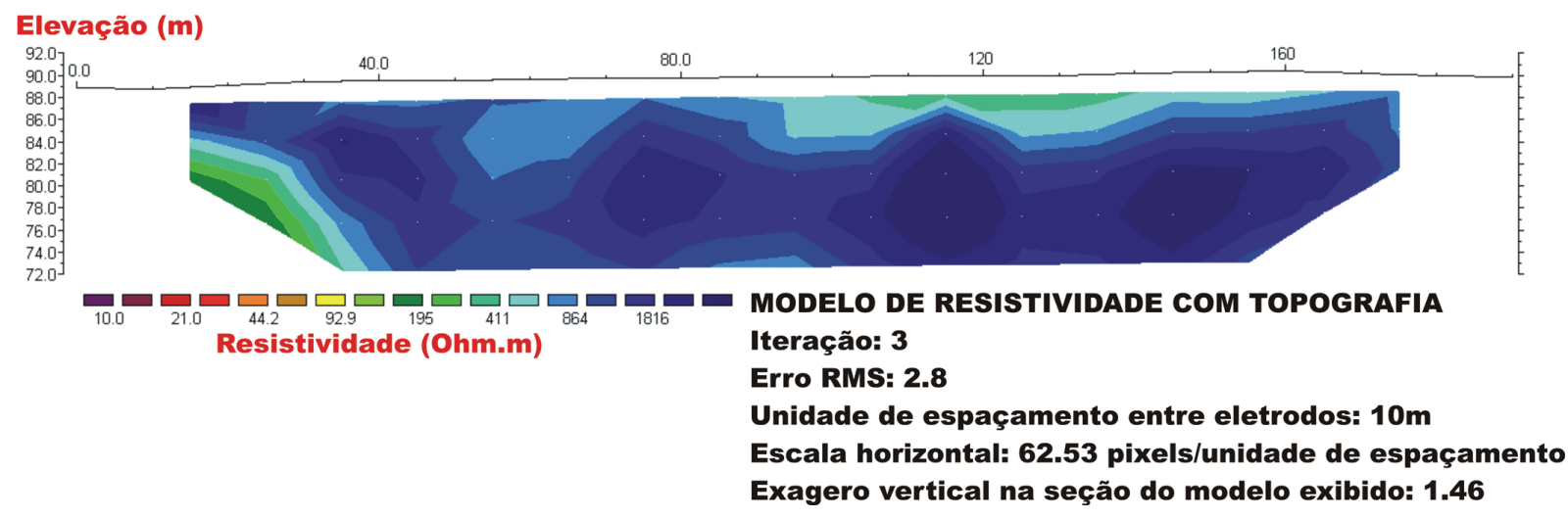

Figura 12 - Linha TQ-CS: 0 início desta linha está a 54 metros de distância da linha TQ-10 e o final desta linha dista 105 metros de TQ-10. 


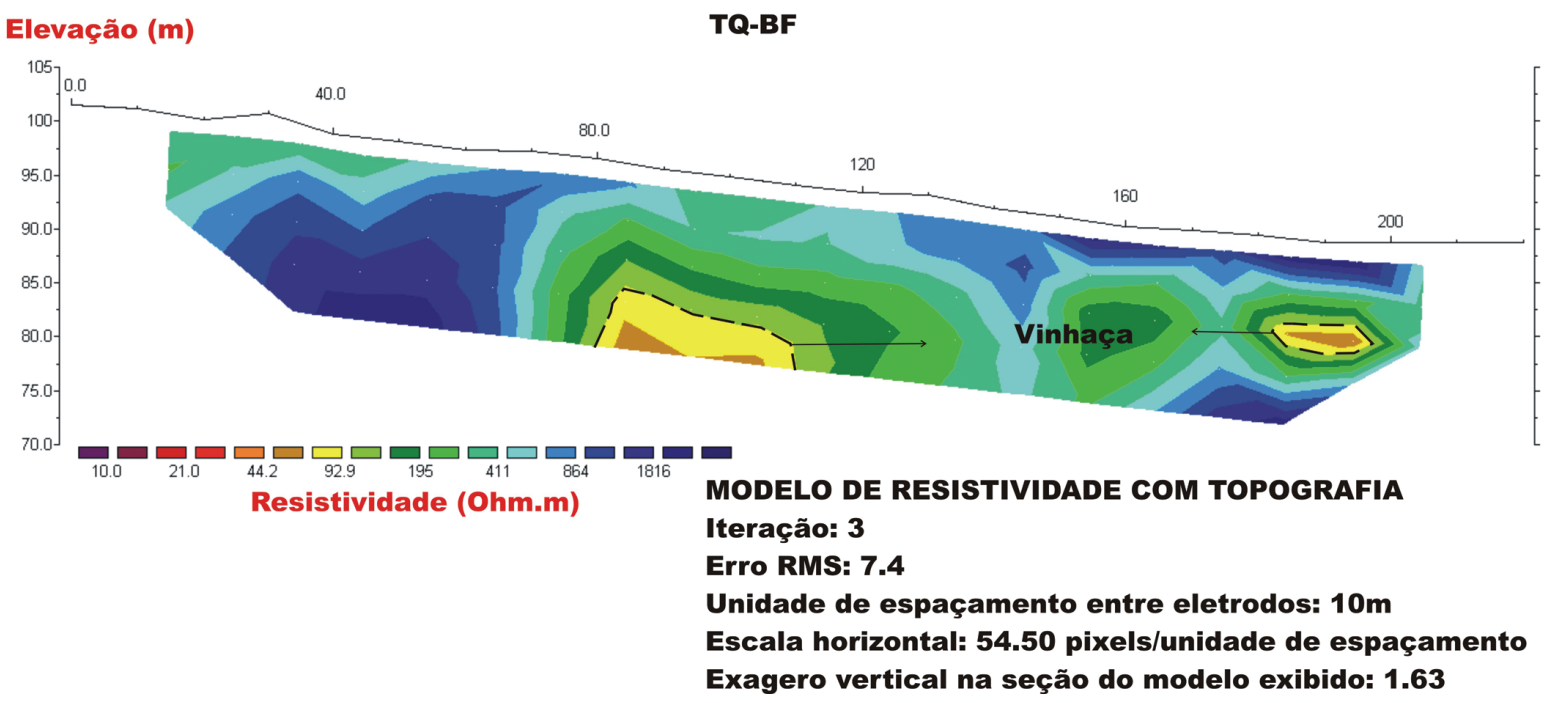

Figura 13 - Linha TQ-BF, localizada na borda lateral externa do tanque. Esta linha corta todas as outras linhas e possui 220 metros de comprimento.

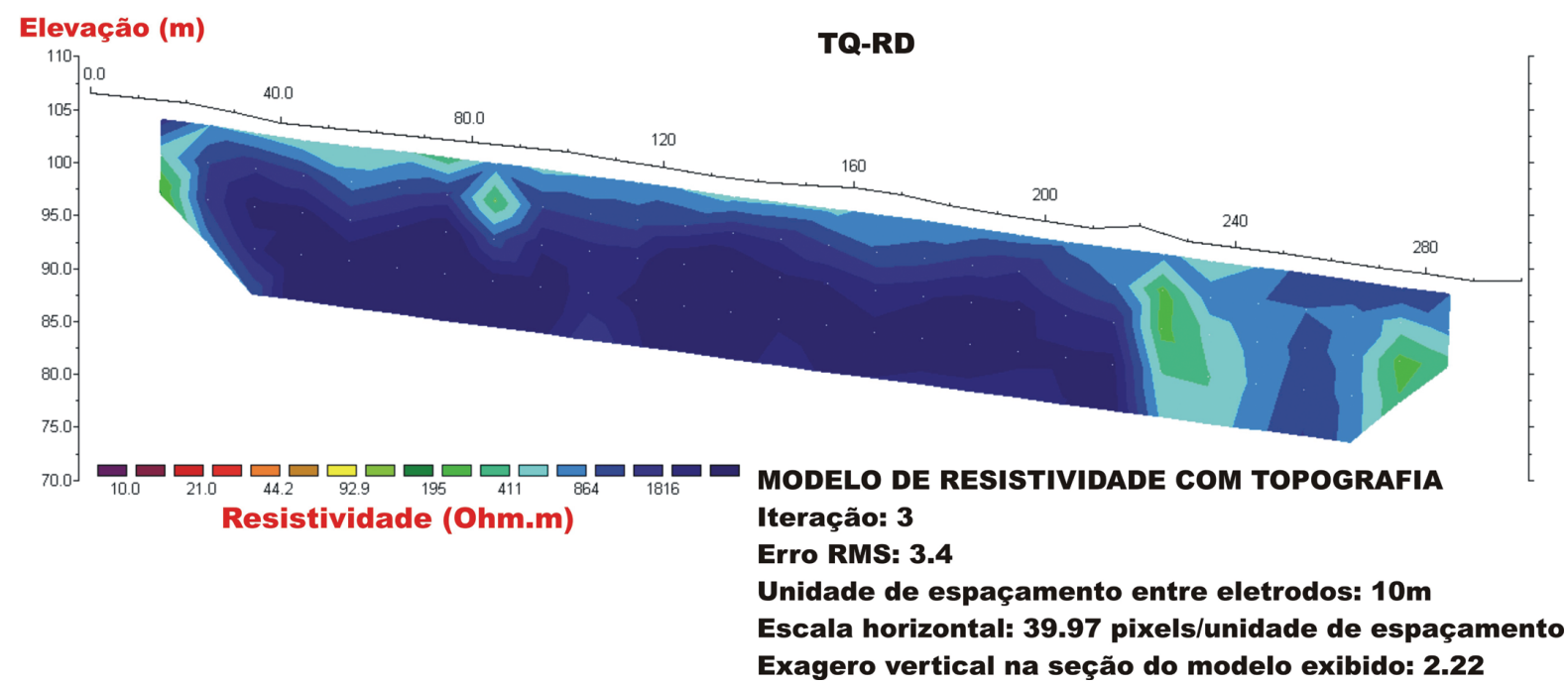

Figura 14 - Linha TQ-RD, possui 300 metros de comprimento e limita a área de influência do contaminante.

As seções apresentadas possuem coerência matemática, são racionais dentro do conjunto de dados adquiridos e se adequam às percepções de campo e aos conhecimentos que se detêm da área.

\section{CONCLUSÕES}

0 método da eletrorresistividade mostrou-se adequado para detectar e mapear a influência da vinhaça no solo.

Os levantamentos eletrorresistivos mostraram um aumento da condutividade elétrica dentro da área do antigo tanque de disposição de vinhoto e a zona de influência do resíduo, bem como altos valores de resistividade para as zonas sem influência da vinhaça.
A influência da vinhaça pode ser caracterizada por baixos vaIores de resistividade entre 10 0hm.m e 90 0hm.m.

Os baixos valores de resistividade encontrados dentro do tanque podem ser atribuídos à influência dos sais presentes na vinhaça e à retenção de água em solo argiloso intensificada pelas forças matriciais e pela grande quantidade de matéria orgânica presente no contaminante. Em contrapartida, os altos valores de resistividade sugerem ausência de influência do contaminante e podem ser atribuídos à argila seca.

As linhas TQ-1, TQ-2, TQ-3, TQ-8, TQ-9 mostraram grande influência da vinhaça no solo, permitindo mapeá-la e permitindo também comprovar a eficiência do método por comparação/contraste com as áreas não contaminadas (TQ-CS e TQ-RD). 
As linhas TQ-10 e TQ-BF sugeriram grande influência da cava na contaminação do solo e permitiram concluir que a contaminação extrapola os limites do tanque; a linha TQ-1A sugeriu baixa influência do resíduo no último nível de investigação.

As linhas TQ-CS e TQ-RD não apontaram anomalias significativas de condutividade no solo e permitiram delimitar a zona de influência do contaminante.

\section{AGRADECIMENTOS}

Os autores agradecem ao CNPq pelo apoio financeiro concedido para a realização deste projeto de pesquisa, à EMBRAPA e ao INCRA pela parceria e pelo apoio técnico. Ao Departamento de Geofísica do Instituto de Astronomia, Geofísica e Ciências Atmosféricas pelo empréstimo dos equipamentos. Ao colega Ivan Mamede e ao técnico Ernande Costa Santos, pelo auxílio nas atividades de campo e a professora Sueli Yoshinaga pela ajuda prestada.

\section{REFERÊNCIAS}

ABU-HASSANEIN ZS, BENSON CH \& BLOTZ LR. 1996. Electrical resistivity of compacted clays. Journal of Geotechnical Engineering, 397-406.

AHMED AM \& SULAIMAN WN. 2001. Evaluation of Groundwater and Soil Pollution in a Landfill Area using Electrical Resistivity Imaging Survey. Environment Management, 5: 28: 655-663.

ARISTODEMOU E \& THOMAS-BETTS A. 2000. DC resistivity and induced polarisation investigations at a waste disposal site and its environments. Journal of Applied Geophysics, 44: 275-302.

BERNSTONE C, DAHLIN T, OHLSSON T \& HOGLAND W. 2000. DCresistivity mapping of internal landfill structures: two pre-excavation surveys. Environmental Geology, 39(3-4): 360-371.

CABRINI MF \& MARJOTTA-MAISTRO MC. 2007. Mercado Internacional de Álcool: os Recentes Programas de Uso do produto como Combustível. Agroanalysis, p. 36.

CETESB (Companhia de Tecnologia de Saneamento Ambiental). 2004. Relatório de qualidade das águas subterrâneas no Estado de São Paulo 2001-2003. São Paulo Série Relatórios/CETESB, 203 p. il.

DER/SP (Departamento de Estradas de Rodagem do Estado de São Paulo). 2006. Duplicação e melhoramentos da pista existente da rodovia SP-333 - Abrão Assed, do km 33,8 ao km 54,8, entre os Municípios de Ribeirão Preto e Serrana. Relatório Ambiental Preliminar - RAP. Disponível em: < www.cmdca.ribeiraopreto.sp.gov.br/splan/ comdema/ aero/ abraoassed.pdf>. Acesso em: 25 jun. 2007.

FREIRE WJ. 2000. Vinhaça de cana-de-açúcar. Série Engenharia Agrícola, vol. 1. $204 \mathrm{p}$.

GLOEDEN E. 1994. Monitoramento da qualidade da água das zonas nãosaturada e saturada em área de fertirrigação com vinhaça. Tese (Mes- trado) - Programa de Pós-Graduação em Recursos Minerais e Hidrogeologia, Universidade de São Paulo (USP). 164 p.

GOLDEN SOFTWARE INC. 2006. Surfer 8.0 for Windows. User's Guide. $340 \mathrm{p}$.

HASSUDA S. 1989. Impactos da infiltração da vinhaça de cana no Aqüífero Bauru. São Paulo, Tese de Mestrado. Instituto de Geociências. Universidade de São Paulo. 92 p.

KELLER GV \& FRISCHKNECHT FC. 1966. Electrical methods in geophysical prospecting. International series of monographs in electromagnetic waves. Pergamon Press, first edition, Vol. 10. 519 p.

LOKE MH. 2000. Electrical imaging surveys for environmental and engineering studies - A practical guide to 2-D and 3-D surveys. Disponível em: <http://www.geoelectrical.com>. Acesso em: 29 jun. 2007.

LUDOVICE MTF. 1997. Estudo do efeito poluente da vinhaça infiltrada em canal condutor de terra sobre lençol freático. 1997. Tese (Mestrado em Engenharia Civil) - Programa de Pós-Graduação em Engenharia Civil, Universidade Estadual de Campinas (UNICAMP). 143 p.

MACEDO IC. 2007. Situação atual e perspectivas do etanol. Estudos Avançados, 21(59): 157-165.

MCNEILL JD. 1980. Electrical conductivity of soils and rocks. Technical Note TN-5. Geonics Limited. $20 \mathrm{p}$.

MEJU MA. 2000. Geoelectrical investigation of old/abandoned, covered landfill sites in urban areas: model development with genetic diagnosis approach. Journal of Applied Geophysics, 144: 115-150.

MENDES JMB. 1987. Técnica geofísicas aplicadas no mapeamento e monitoramento de poluição e contaminação de águas subterrâneas. Tese (Doutorado em Geociências). Universidade de São Paulo (USP). 196 p.

MOURA HP \& MALAGUTTI FILHO W. 2003. Métodos de eletrorresistividade e de polarização induzida aplicados na área de disposição de resíduos urbanos: aterro controlado de Rio Claro - SP. UNESP, Geociências, $22\left(n^{\circ}\right.$ especial): 129-139.

PARKHOMENKO El. 1967. Electrical properties of rocks. KELLER GV (Ed.). Translated from russian, Plenum Press, New York, NY, 314 p.

REYNOLDS JM. 1997. An introduction to applied and environmental geophysics. Ed. John Wiley and Sons. 796 p.

UNICA (União da Agroindústria Canavieira de São Paulo). 2007. Produção e uso do etanol combustível no Brasil. Respostas às questões mais freqüentes. São Paulo. 70 p.

VAN DAM RL. 2001. Causes of ground-penetrating radar reflections in sediment. Amsterdam: Vrije Universiteit, Faculty of Earth Sciences, Doctoral Thesis, $110 \mathrm{p}$.

VOGELSANG D. 1995. Environmental geophysics - A practical guide. Springer-Verlag, New York, $173 \mathrm{p}$. 


\section{NOTAS SOBRE OS AUTORES}

Juliana Igarashi da Cruz. Formada em Tecnologia em Controle e Saneamento Ambiental pela Universidade Estadual de Campinas, UNICAMP, 2006. Mestre pelo Programa de Pós-Graduação em Geociências do IGe/UNICAMP com estudos complementares na Universidad Complutense de Madrid (UCM). Atua principalmente nas áreas de contaminação do solo e métodos geoelétricos.

Maria Carmen Hernández Lucendo. Doutora em Ciências Físicas (especialidade Geofísica) pela Universidad Complutense de Madrid em 1982. Desde 1984 e professora titular da universidade no Departamento de Física de la Tierra I da Faculdad de Ciencias Físicas (UCM). Responsável pelo grupo de Exploração Geofísica no departamento e utiliza fundamentalmente os métodos elétricos em corrente continua, GPR, magnético e sísmico para o estudo da zona mais superficial da crosta terrestre. Desde 1986 trabalha na aplicação de métodos geofísicos aplicados ao estudo de jazidas arqueológicas e de patrimônio histórico.

Rodrigo de Souza Portugal. Possui graduação em Matemática Aplicada e Computacional pela Universidade Estadual de Campinas (1995), mestrado em Matemática Aplicada pela Universidade Estadual de Campinas (1998) e doutorado em Matemática Aplicada pela Universidade Estadual de Campinas (2002). Atualmente é professor doutor do Departamento de Geologia e Recursos Naturais do Instituto de Geociências da Universidade Estadual de Campinas. Tem experiência na área de Geociências, com ênfase em Geofísica Aplicada, atuando principalmente nos temas relacionados à teoria do imageamento sísmico, processamento de imagens geofísicas, caracterização de reservatórios, geofísica matemática e matemática aplicada à geologia.

Vagner Roberto Elis. Graduado em Geologia em 1990 pela Universidade Estadual Paulista, Mestre em Geociências pela Universidade Estadual Paulista em 1993 desenvolvendo a dissertação "A aplicação da geofísica para análise do meio físico. Importância para elaboração de mapeamento geotécnico". Doutor em Geociências pela Universidade Estadual Paulista em 1999 defendendo a tese "Avaliação da aplicabilidade de métodos elétricos de prospecção geofísica no estudo de áreas utilizadas para disposição de resíduos". Docente do Departamento de Geofísica do Instituto de Astronomia, Geofísica e Ciências Atmosféricas da Universidade de São Paulo, com atuação nas áreas de Geofísica Aplicada a estudos ambientais e hidrogeológicos.

Sergio Junior da Silva Fachin. Bacharel em Geologia (2005) pelo Instituto de Ciências Exatas e da Terra da Universidade Federal de Mato Grosso (ICET/UFMT). Mestre em Ciências - Área: Geofísica (2007) pelo Instituto de Astronomia, Geofísica e Ciências Atmosféricas da Universidade de São Paulo (IAG/USP) com o trabalho intitulado "Ensaios geoelétricos 2D no antigo lixão de Ribeirão Preto - SP: avaliação de parâmetros de aquisição e monitoramento ambiental do problema". Atualmente atua com a prestação de serviços em geologia e geofísica aplicada a mineração, geotecnia, hidrogeologia e problemas ambientais.

Andréa Teixeira Ustra. Formada em Física pela Universidade Estadual de Campinas, UNICAMP, 2004. Atualmente é aluna de mestrado do Programa de PósGraduação em Geofísica do IAG/USP, desenvolvendo a pesquisa "Caracterização geoelétrica de uma área contaminada à jusante do Aterro de Resíduos Sólidos Urbanos de Bauru-SP".

Welitom Rodrigues Borges. Graduado em Geologia pela Universidade Federal de Mato Grosso (2000), concluiu 0 mestrado (2002) e 0 doutorado (2007) em Geofísica Aplicada no Instituto de Geofísica, Astronomia e Ciências Atmosféricas (IAG) da Universidade de São Paulo. Consultor em processamento de dados GPR no convênio entre a Unicamp e a empresa Brain Tecnologia (2004-2006). Consultor em GPR na empresa SIGEO - Soluções Integradas em Geotecnologias (2005-2007). Consultor em geofísica na empresa Geopesquisa Investigações Geológicas (2006-2007). Atua em projetos geofísicos de identificação de resíduos, interferências no solo, água subterrânea e depósitos minerais. 